\section{Active Limb Activation}

Sarah A. Raskin

Department of Psychology and Neuroscience

Program, Trinity College, Hartford, CT, USA

\section{Synonyms}

Limb activation

\section{Definition}

Active limb activation is a rehabilitation technique for individuals with unilateral neglect. In a series of studies, Robertson and North (1992, 1993, 1994) and others (Mattingly et al. 1998) have demonstrated that moving the upper or lower extremity on the affected side can reduce neglect symptoms. The effect is seen only with active movement, as opposed to passive movement, and only when the limb is moved in the effected hemispace. However, the limb need not be observed visually. It should be noted that the effect has not been demonstrated universally (e.g., Brown et al. 1999), and treatment effects may be specific to movements in peripersonal space (Priftis et al. 2013).

\section{Cross-References}

- Attention Training

- Behavioral Inattention Test

- Cognitive Rehabilitation

- Neglect Syndrome

\title{
References and Readings
}

Brown, V., Walker, R., Gray, C., \& Findlay, J. (1999). Limb activation and the rehabilitation of unilateral neglect: Evidence of task-specific effects. Neurocase, 5, 129-142.

Mattingly, J., Robertson, I., \& Driver, J. (1998). Modulation of covert visual attention by hand movement: Evidence from parietal extinction after right hemisphere damage. Neurocase, 4, 245-253.

Priftis, K., Passarini, L., Pilosio, C., Meneghello, F., \& Pitteri, M. (2013). Visual scanning training, limb activation treatment, and prism adaptation for rehabilitating left neglect: Who is the winner? Frontiers in Human Neuroscience, 7, 1-12.

Robertson, I., \& North, N. (1992). Spatio-motor cueing in unilateral left neglect: The role of hemispace, hand and motor activation. Neuropsychologia, 30, 553-563.

Robertson, I., \& North, N. (1993). Active and passive activation of left limbs: Influence on visual and sensory neglect. Neuropsychologia, 31, 293-300.

Robertson, I., \& North, N. (1994). One hand is better than two: Motor extinction of left hand advantage in unilateral neglect. Neuropsychologia, 32, 1-11. 